\title{
Rheology of Confined Non-Brownian Suspensions
}

\author{
Walter Fornari, ${ }^{1}$ Luca Brandt, ${ }^{1}$ Pinaki Chaudhuri, ${ }^{2}$ Cyan Umbert Lopez, ${ }^{1}$ Dhrubaditya Mitra, ${ }^{3}$ and Francesco Picano ${ }^{4}$ \\ ${ }^{1}$ Linné Flow Centre and SeRC, KTH Mechanics, SE-100 44 Stockholm, Sweden \\ ${ }^{2}$ Institute of Mathematical Sciences, CIT Campus, Taramani, Chennai 600113, India \\ ${ }^{3}$ Nordita, KTH Royal Institute of Technology and Stockholm University, Roslagstullsbacken 23, 10691 Stockholm, Sweden \\ ${ }^{4}$ Department of Industrial Engineering, University of Padova, Via Venezia 1, 35131 Padova, Italy
}

(Received 24 June 2015; published 8 January 2016)

\begin{abstract}
We study the rheology of confined suspensions of neutrally buoyant rigid monodisperse spheres in plane-Couette flow using direct numerical simulations. We find that if the width of the channel is a (small) integer multiple of the sphere diameter, the spheres self-organize into two-dimensional layers that slide on each other and the effective viscosity of the suspension is significantly reduced. Each two-dimensional layer is found to be structurally liquidlike but its dynamics is frozen in time.
\end{abstract}

DOI: 10.1103/PhysRevLett.116.018301

Suspensions of solid objects in simple Newtonian solvents (e.g., water) can show a kaleidoscope of rheological behaviors depending on the shape, size, and volume fraction $(\phi)$ of the additives, and the shear rate $(\dot{\gamma})$ imposed on the flow; see, e.g., Refs. [1,2] for reviews. Suspensions can be of various types; e.g., suspensions of small particles (smaller than the viscous scale of the solvent), where the solvent plays the role of a thermal bath, are called Brownian suspensions (e.g., colloids) [1]. At a small $\phi$ and under a small $\dot{\gamma}$, the effective viscosity of such suspensions increases with $\phi: \mu_{\text {eff }} \sim(5 / 2) \phi$ [3], as derived by Einstein [4] (See also Ref. [5] for a $d$ dimensional generalization.) In the other category are suspensions of large particles (e.g., emulsion, granular fluids, etc.), where the thermal fluctuations are often negligible. Such suspensions are called non-Brownian suspensions. For moderate values of $\phi$ and $\dot{\gamma}$, non-Brownian suspensions show continuous shear thickening (i.e., $\mu_{\text {eff }}$ increases with $\dot{\gamma}$ ) [6,7], which can be understood [7] in terms of excluded volume effects. Such a rheological response has been observed in many natural and industrial flows, including flows of mud, lava, cement, and corn-starch solutions. Dense (large $\phi$, close to random close packing) non-Brownian suspensions may show discontinuous shear thickening [8,9]—a jump in effective viscosity as a function of $\dot{\gamma}$.

Recent experiments have uncovered intriguing rheological behavior of dense (large $\phi$ ) suspensions under confinement $[6,8,10]$. Common wisdom suggests that, under confinement, the inertial effects are generally unimportant at small $\dot{\gamma}$ 's. However, a series of recent studies [11-14] have demonstrated that the effect of fluid inertia, although small, can give rise to a variety of effects even in microfluidic flows. Drawing inspirations from these two sets of works, we study the effects of confinement on nonBrownian suspensions with moderate values of $\phi$ and $\dot{\gamma}$. In particular, we choose the range of $\phi$ and $\dot{\gamma}$ such that, in bulk, the suspensions show continuous shear thickening.
As a concrete example, we consider direct numerical simulations of three-dimensional plane-Couette flow-with the $x, y$, and $z$ coordinates along the streamwise, spanwise, and wall-normal directions (see Fig. 1 of the Supplemental Material [15]) seeded with neutrally buoyant rigid spheres of radius $a$. The fluid is sheared by imposing constant streamwise velocity of opposite signs, $U_{0}=\dot{\gamma} L_{z} / 2$ at $z= \pm L_{z} / 2$. We study the effects of confinement by changing the dimensionless ratio $\xi \equiv L_{z} / 2 a$, where $L_{z}$ is the channel width in the $z$ direction.

The most striking result of our simulations is that at or near integer values of $\xi$, the effective viscosity $\mu_{\text {eff }}$ decreases significantly compared to its bulk value [Figs. 1(a) and 1(b)]. This drop can be so large that the volumetric flow rate in a thinner channel $(\xi=2)$ is more than in a wider channel $(\xi=2.5)$; see Fig. 1(c). We further demonstrate that, at small integer values of $\xi$, the rigid spheres self-organize into an integer number of horizontal layers, which slide on one another; see Fig. 2. This, in turn, decreases the transport of horizontal momentum across the layers generating the drop in effective viscosity. We also show that at integer values of $\xi$, for which layers appear, the following occurs: (i) the movement of spheres across layers is not a diffusive process (see Fig. 3); (ii) the typical residence time of the spheres in a layer is much longer than the time scale set by the shear, $\dot{\gamma}^{-1}$; in fact, a large number of spheres never leave the layer within the runtime of the simulations [see Fig. 4(a)]; (iii) the layers are structurally liquidlike but dynamically very slow compared to the time scale set by $\dot{\gamma}^{-1}$ (see Fig. 4).

In the numerical simulations, the fluid phase is described by solving the incompressible Navier-Stokes equations in three dimensions. Periodic boundary conditions are imposed on the two directions ( $x$ and $y$, with lengths $L_{x}$ and $L_{y}$, respectively). The motion of the rigid spheres and their interaction with the flow is fully resolved by using the immersed boundary method [16-18]. Lubrication models 

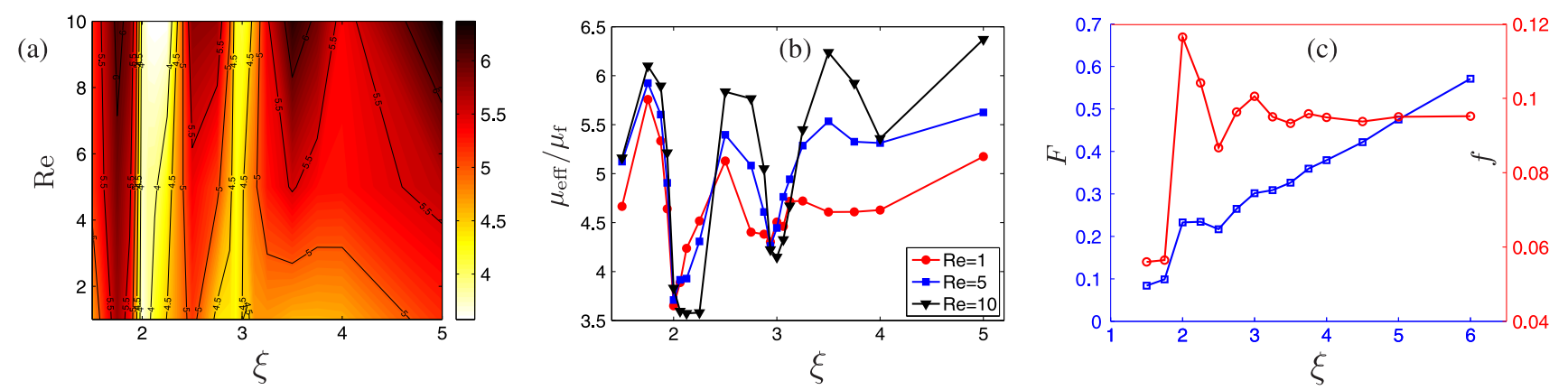

FIG. 1. (a) Contour plot of the effective viscosity $\mu_{\text {eff }} / \mu_{f}$ as a function of $\xi$ and Re for $\phi=0.3$. (b) The effective viscosity $\mu_{\text {eff }} / \mu_{f}$ vs $\xi$ for $\phi=0.3$ and for three different values of Re: $\mathrm{Re}=1$ (red circles), 5 (blue squares), and 10 (black triangles). (b) The flow rate of matter (both solvent and additive) $F$ (blue squares, left vertical axis), defined in Eq. (1), and the flow-rate-per-unit cross section $f$ (red circles, right vertical axis) as a function of $\xi$, for $\operatorname{Re}=5$ and $\phi=0.3$.

based on Brenner's analytical solution and a soft-sphere collision model are used to properly reproduce the interactions among particles when the particle distance is below the mesh size [19]. A complete description of the equations and details of the algorithm are provided in the Supplemental Material [15].

In practice, we change $L_{z}$ but hold the particle radius $a$ fixed. The effective viscosity, $\mu_{\text {eff }}$, is thus a function of the dimensionless numbers, $\phi$ and $\xi$, and the particle Reynolds number, $\operatorname{Re} \equiv \rho \dot{\gamma} a^{2} / \mu_{f}$, where $\rho$ and $\mu_{f}$ are the density and dynamic viscosity of the solvent. The size of the rectangular box is $16 a \times 16 a$ in the streamwise and spanwise directions. The Cartesian mesh uses eight grid points per particle radius $a$. The surface of each particle is discretized with 746 Lagrangian grid points.

Initially, the spheres are placed at random locations, with no overlap between each other, and with velocities equal to the local fluid velocity of the laminar Couette profile (see Fig. 1 of the Supplemental Material [15]). We calculate the effective viscosity, $\mu_{\text {eff }}$, as the ratio between the tangential stress at the walls and the shear $\dot{\gamma}$ averaged over space and time. See the Supplemental Material [15] for further details of measurement and an estimate of errors.

The effective viscosity $\mu_{\text {eff }}$ is shown in Figs. 1(a) and 1(b) as a function of $\operatorname{Re}$ and $\xi$, for $\phi=0.3$. For large channel widths, e.g., $\xi=5$, and large volume fractions, e.g., $\phi \geq 0.2$, we obtain shear thickening (see the Supplemental Material, Fig. 4 [15]), as was found earlier in Ref. [7].

Here, we address how confinement affects the rheology. Experiments [20], in agreement with earlier numerical calculations [21], have found that at a small volume fraction $(\phi \leq 0.15), \mu_{\text {eff }}$ increases as $\xi$ decreases. Our results (see the Supplemental Material, Fig. 4 [15]) also support this conclusion. Furthermore, our simulations can access hitherto unstudied higher values of the volume fraction $(\phi \geq 0.2)$ for which this trend seems to reverse; i.e., $\mu_{\text {eff }}$ decreases with confinement. Upon deeper scrutiny, a more striking picture emerges. As we show in Fig. 1(b), for $\phi=0.3$, at or near small integer values of $\xi(\xi=2,3$, and 4), the effective viscosity drops significantly. The minimum value of viscosity is found at $\xi=2$, which is as low as $50 \%$ of its bulk value ( $\xi \geq 6$ ). To appreciate how dramatic this effect is, we measure the (dimensionless) flux of matter (i.e., both the fluid and the spheres) through the channel, defined as

$$
F \equiv \frac{1}{\dot{\gamma} L_{z} a^{2}}\left\langle\int V_{x} \operatorname{sgn}(z) d y d z\right\rangle,
$$
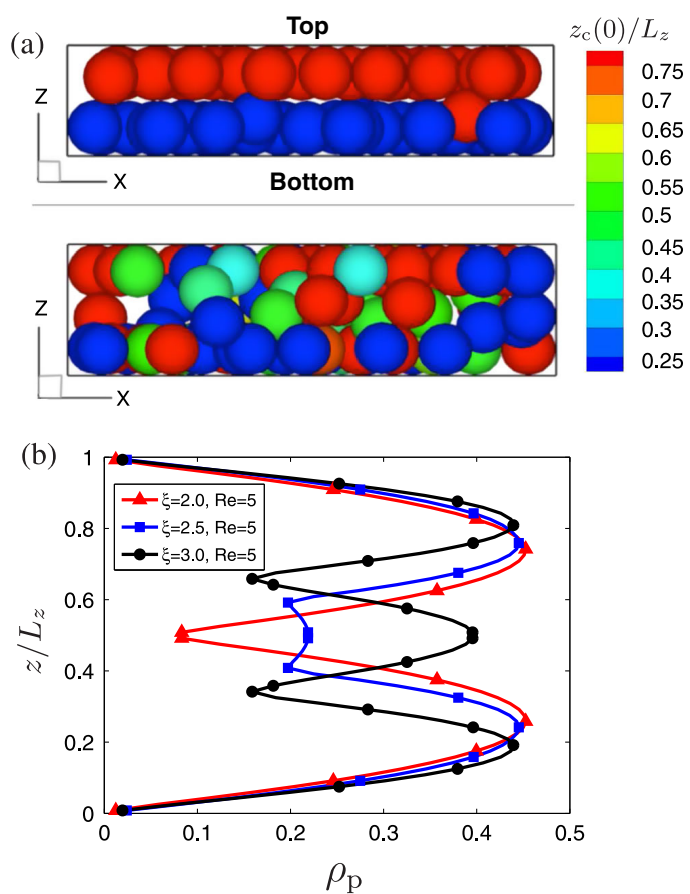

FIG. 2. (a) Three-dimensional view of positions of the spheres for $\operatorname{Re}=5, \phi=0.3$, and $\xi=2$ (top panel) and $\xi=2.5$ (bottom panel) at later times after the initial transients. The particles are color coded by their initial wall-normal position (red, close to the top boundary; blue, close to the bottom boundary). (b) The local average particle volume fraction, $\rho_{p}$, vs the wall-normal coordinate $z$, for $\xi=2$ (red triangles), 2.5 (blue squares), and 3 (black dots). 
where $V \equiv \zeta U^{p}+(1-\zeta) u$ [22], with $\zeta=1$ at a grid point inside a rigid sphere but $\zeta=0$ otherwise [23]. Here, $U^{p}$ is the velocity of the sphere, $u$ is the velocity of the fluid and $\langle\cdots\rangle$ denotes an averaging over time. In Fig. 1(c) we report $F$ as a function of $\xi$. As the flux $F$ is not normalized by the cross-sectional area of the channel, it is expected to increase linearly as a function of $\xi$. This expectation indeed holds for $\xi>4$. But, below that, for integer values of $\xi$ the effective viscosity can decrease so much that $F$ is not even a monotonic function of $\xi$-in particular, $F(\xi=2)>F(\xi=2.5)$; the flux through a wider channel is actually smaller. The flux per unit area shown in Fig. 1(c), $f \equiv F a^{2} /\left(L_{y} L_{z}\right)$, is expected to be a constant at large $\xi$ 's. For the small integer values of $\xi$, it is significantly higher than its bulk value.

The drop in $\mu_{\text {eff }}$ at integer values of $\xi$ was experimentally observed in Ref. [10], whose authors studied the problem in a different parameter regime-negligible inertia and high volume fraction-where the nonconfined solution shows discontinious shear thickening. Other experimental [20] and numerical work [21], again in the realm of negligible inertia, also observed some signatures of this intriguing phenomenon [24], which, according to our results, is an effect of inertia missing from these earlier works.

To investigate the mechanism behind the rheology, we examine snapshots of the spheres; see Fig. 2(a) for $\xi=2$ (top panel) and $\xi=2.5$ (bottom panel). The spheres are color coded by their initial wall-normal locations (red corresponds to an initial position near the top boundary and blue to the bottom boundary). It can be clearly observed that, for $\xi=2$, the particles form a bilayered structure. This layering is also confirmed by the wallnormal profiles of the average particle volume fraction, $\rho_{p}(z) \equiv\langle\zeta(x, y, z, t)\rangle_{x, y, t}$, displayed in Fig. 2(b) for $\xi=2,2.5$, and 3 . In the first and the last case, one can observe equally spaced two and three prominent peaks, respectively. Note that a weaker layering is observed for $\xi=2.5$. The drop in effective viscosity thus corresponds to the formation of layers that slide on each other, with little transport of momentum across the layers. For $\xi=2$, where layering is most prominent, the particles form disordered liquidlike structures within each layer, as seen by the radial distribution function of the position of the spheres; see the Supplemental Material, Fig. 7 [15]. Within a layer, each sphere does not simply slide with the flow-it is also shows rolling; see the Supplemental Material, Fig. 9 [15].

In order to understand the dynamics of particles in the wall-normal direction, we show in Fig. 3(a) the probability distribution function (PDF) of the displacement of the spheres, $d_{z}(t) \equiv z_{c}(t)-z_{c}(0)$, at different times, where $z_{c}(t)$ is the the $z$ coordinate of the center of a sphere. Obviously, as $t \rightarrow 0$ the PDF $\left[P\left(d_{z}\right)\right]$ must approach a Dirac delta function. Remarkably, for $\xi=2$ the PDF has exponential tails; i.e., it is nondiffusive, with some particles undergoing larger displacements as shown in Fig. 3(a).
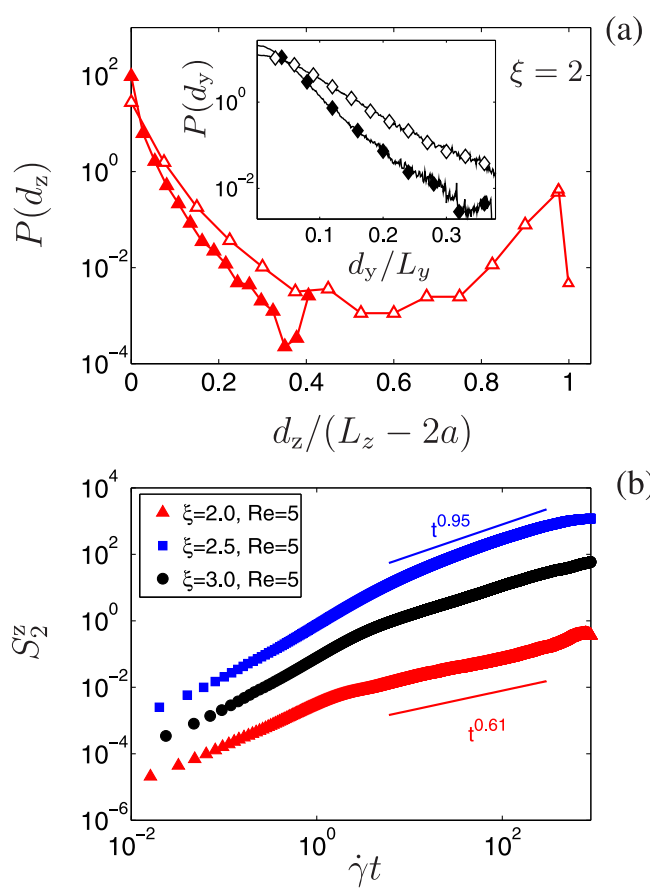

(b)

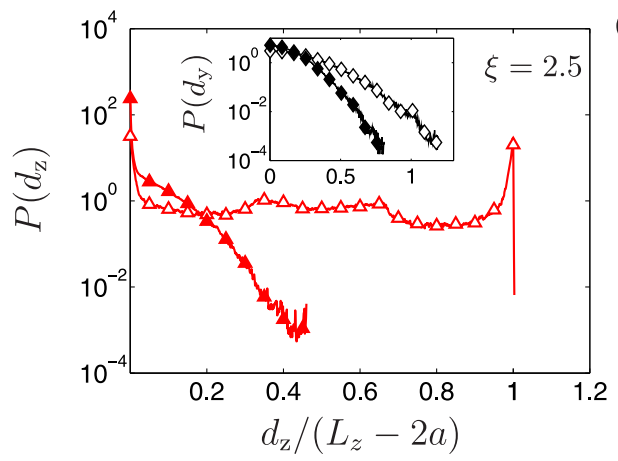

(c)

FIG. 3. (a) The PDF $P\left(d_{z}\right)$ of the displacement $\left(d_{z}\right)$ of the center of the spheres along the $z$ direction for $\xi=2$, at early times, $t=2.5 \dot{\gamma}^{-1}$ (red filled triangles) and late times $t=386 \dot{\gamma}^{-1}$ (red open triangles) for $\mathrm{Re}=5$. For comparison, the inset shows the PDF $P\left(d_{y}\right)$ of the displacement $\left(d_{y}\right)$ along the $y$ direction at early times, $t=2.5 \dot{\gamma}^{-1}$ (black filled diamonds) and moderate times $t=5 \dot{\gamma}^{-1}$ (black open diamonds). (b) Log-log plot of mean squared displacement of the spheres $S_{2}^{z}$ vs $t \dot{\gamma}$. The curves have been translated in the $y$ direction for clarity. At late times, $S_{2}^{z} \sim t^{\beta}$, where $\beta=1$ implies diffusion. For three different values of $\xi=2$ (red triangles), 2.5 (blue squares), and 3 (black dots), we obtain $\beta \approx 0.61,0.95$, and 0.88 , respectively. (c) The PDF $P\left(d_{z}\right)$ of the displacement $\left(d_{z}\right)$ of the center of the spheres along the $z$ direction for $\xi=2.5$. The PDF of $d_{y}, P\left(d_{y}\right)$, is shown in the inset.

At later times, for $\xi=2$, the PDF of $d_{z}$ develops a peak at $d_{z} /\left(L_{z}-2 a\right)=1$, indicating the hop between two layers. A similar behavior is observed for $\xi=3$, too. Contrast this result with the PDF of the displacement along the spanwise $(y)$ direction [see the inset of Fig. 3(a)], which clearly shows Gaussian behavior at all times, implying diffusive dynamics. The second moment of these PDFs provides the mean squared displacement of the particles, 

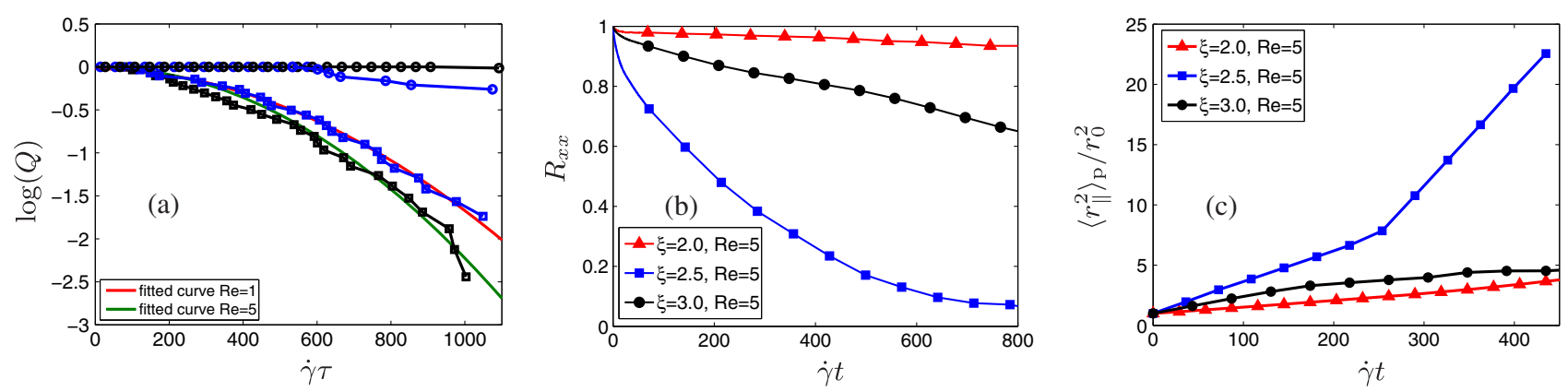

FIG. 4. (a) Cumulative PDF $Q$ of the residence times of spheres $(\dot{\gamma} \tau)$ in a layer for $\phi=0.3, \operatorname{Re}=1$ (in blue), and $\operatorname{Re}=5$ (in black), and $\xi=2$ (circles) and $\xi=2.5$ (squares). (b) The streamwise velocity-velocity autocorrelation function $R_{x x}$ for $\operatorname{Re}=5$ and $\phi=0.3$ for four different values of $\xi=2$ (red triangles), 2.5 (blue squares), and 3 (black dots). (c) Evolution of the averaged square of relative distance between pairs of sphere $\left\langle r_{\|}^{2}\left(t, r_{0}\right)\right\rangle_{p}$, which were at distance $r_{0}$ at $t=0$, for $r_{0}=4 a$, for three different values: $\xi=2$ (red triangles), 2.5 (blue squares), and 3 (black dots).

$S_{2}^{z}(t)=\left\langle\left[z_{c}(t)-z_{c}(0)\right]^{2}\right\rangle_{p}$, the time evolution of which is shown in Fig. 3(b). At late times, in general, a power-law dependence on time is found, $S_{2}^{z}(t) \sim t^{\beta}$, where $\beta=1$ would imply a simple diffusive behavior. For $\xi=2,3$ we estimate $\beta \approx 0.61$ and 0.88 , respectively. But for $\xi=2.5$, $\beta \approx 1$ is obtained. The diffusive behavior for $\xi=2.5$ can be further confirmed by plotting the PDF of $d_{z}$. At early times $\left(t=2.5 \dot{\gamma}^{-1}\right)$, for $\xi=2.5$, the PDF develops Gaussian tails - indicative of a diffusive process - and at late times it approaches a constant [see Fig. 3(c)].

To display the dynamics, we provide movies of the particles' trajectories in the Supplemental Material [15]. These clearly demonstrate that for $\xi=2$, each horizontal layer is structurally disordered but dynamically frozen (i.e., the particle motion relative to the layer is dynamically frozen). We quantify this phenomenon by three different measurements. (A) We calculate the residence time $(\tau)$ of a sphere in a single layer. The residence time is defined as $\tau=\max \left\{t \mid d_{z}(t)< \pm a\right\}$. Instead of calculating the PDF by histograms of $\tau$, we calculate the cumulative PDF, $Q(\tau)$, by the rank-order method [25], as the latter is free from binning errors. The cumulative PDF, $Q(\tau)$, is displayed in Fig. 4(a) as a function of $\tau$ for $\xi=2$ and 2.5 for $\operatorname{Re}=1$ and 5 . For $\xi=2, Q$ remains very close to unity during the whole duration of the simulation; i.e., very few spheres actually move from one layer to another. In other words, the layers are quite stable to perturbations in wallnormal directions. Conversely, for $\xi=2.5, Q(\tau)$ can be fitted by a Gaussian. (B) The streamwise velocity autocorrelation function of the spheres, $R_{x x} \equiv\left\langle U_{x}^{p}(t) U_{x}^{p}(0)\right\rangle$, is shown in Fig. 4(b). For $\xi=2, R_{x x} \approx 1$ for a very long time, implying that the temporal fluctuations of the streamwise velocity are negligible. This suggests that each sphere moves in a layer with a uniform streamwise velocity, keeping their relative distances practically constant. For the cases where the layering is not very strong, e.g., $\xi=2.5$ the autocorrelation function decays rapidly. For $\xi=3$, layering reappears and $R_{x x}$ again shows slow decay over time. (C) Let us define $r_{\|}\left(t ; r_{0}\right)$ to be the horizontal distance between a pair of spheres at time $t$ which were at a distance $r_{0}$ at $t=0$ (and in the same layer). If the layers formed by the spheres were truly frozen in time, we would obtain $r_{\|}\left(t ; r_{0}\right)=r_{0}$ for all $t$ 's and $r_{0}$ 's. In Fig. 4(c), we show the time evolution of $\left\langle r_{\|}^{2}\left(t ; r_{0}\right)\right\rangle_{p}$, for $r_{0}=4 a$, where the symbol $\langle\cdots\rangle_{p}$ denotes an averaging over all possible particle pairs [26]. Had the spheres moved chaotically within a layer, $r_{\|}$would have grown exponentially with time. Clearly, for all of the cases shown in Fig. 4(c), $r_{\|}$grows, at most, linearly with time. In particular, when layering occurs $(\xi=2,3)$, it takes a long time $\left(t \geq 430 \dot{\gamma}^{-1}\right)$ for $r_{\|}(t ; 4 a)$ to grow by a factor of 2 . This quantifies again the dynamical stability of the layer: the relative in-plane distance between pairs of spheres changes slowly (linearly) with time.

In conclusion, using numerical simulations, we demonstrate that the effective viscosity of an extremely confined non-Brownian suspension can exhibit a nonmonotonic dependence on the channel width; in particular, the effective viscosity sharply decreases if the channel width is a (small) integer multiple of particle diameter. We demonstrate that this behavior is accompanied by a change in microstructure, namely, the formation of particle layers parallel to the confining plates. The two-dimensional layers formed by the particles slide on each other-the layers are structurally liquidlike because they evolve on very slow time scales. Similar layering under shear has been theoretically anticipated [27,28], but the consequences for transport in extreme confinement that we show have never been demonstrated before. We finally note that our results are in contrast with the case of sheared Brownian suspensions, where the structures were found to be uncorrelated with measured viscosity [29]. Note also that recent studies [30,31] have found anomalous rheological behavior in confined suspension of vesicles (and capsules), where the self-organization of the capsules into files is also observed. In such suspensions, the deformability and the nonsphericity of the capsules are also expected to play a role. 
L. B. and W.F. acknowledge financial support through European Research Council Grant No. ERC-2013-CoG616186 , TRITOS, as well as the computer time provided by SNIC (the Swedish National Infrastructure for Computing). D. M. acknowledges financial support from the Swedish Research Council under Grants No. 2011-542 and No. 6382013-9243. P. C. thanks NORDITA for their hospitality.

[1] N. J. Wagner and J. F. Brady, Phys. Today 62, 27 (2009).

[2] J. J. Stickel and R. L. Powell, Annu. Rev. Fluid Mech. 37, 129 (2005).

[3] G. Batchelor, An Introduction to Fluid Dynamics (Cambridge University Press, Cambridge, England, 1967).

[4] A. Einstein, Ann. Phys. (Berlin) 19, 289 (1906).

[5] J. F. Brady, Int. J. Multiphase Flow 10, 113 (1983).

[6] A. Fall, A. Lemaître, F. Bertrand, D. Bonn, and G. Ovarlez, Phys. Rev. Lett. 105, 268303 (2010).

[7] F. Picano, W.-P. Breugem, D. Mitra, and L. Brandt, Phys. Rev. Lett. 111, 098302 (2013).

[8] E. Brown and H. M. Jaeger, Phys. Rev. Lett. 103, 086001 (2009).

[9] N. Fernandez, R. Mani, D. Rinaldi, D. Kadau, M. Mosquet, H. Lombois-Burger, J. Cayer-Barrioz, H. J. Herrmann, N. D. Spencer, and L. Isa, Phys. Rev. Lett. 111, 108301 (2013).

[10] E. Brown, H. Zhang, N. A. Forman, B. W. Maynor, D. E. Betts, J. M. DeSimone, and H. M. Jaeger, J. Rheol. 54, 1023 (2010).

[11] D. Di Carlo, Lab Chip 9, 3038 (2009).

[12] W. Lee, H. Amini, H. A. Stone, and D. Di Carlo, Proc. Natl. Acad. Sci. U.S.A. 107, 22413 (2010).

[13] D. Di Carlo, J. F. Edd, K. J. Humphry, H. A. Stone, and M. Toner, Phys. Rev. Lett. 102, 094503 (2009).

[14] H. Amini, E. Sollier, W. M. Weaver, and D. Di Carlo, Proc. Natl. Acad. Sci. U.S.A. 109, 11593 (2012).

[15] See Supplemental Material at http://link.aps.org/ supplemental/10.1103/PhysRevLett.116.018301 for (a) description of the algorithm used in the simulations, (b) movies that shows the evolution of the spheres (c) additional figures.

[16] C. Peskin, Acta Numer. 11, 479 (2002).

[17] R. Mittal and G. Iaccarino, Annu. Rev. Fluid Mech. 37, 239 (2005).

[18] W.-P. Breugem, J. Comput. Phys. 231, 4469 (2012).

[19] R. A. Lambert, F. Picano, W.-P. Breugem, and L. Brandt, J. Fluid Mech. 733, 528 (2013).

[20] P. Peyla and C. Verdier, Europhys. Lett. 94, 44001 (2011).

[21] Y. Davit and P. Peyla, Europhys. Lett. 83, 64001 (2008).

[22] F. Picano, W.-P. Breugem, and L. Brandt, J. Fluid Mech. 764, 463 (2015).

[23] The phase indicator $\zeta$ is related to the volume fraction by $\phi=(1 / V) \int \zeta d V$, where $V$ is the total volume of the channel.

[24] The experimental data reported in Ref. [20] is for small to moderate values of $\phi$. At the higher value of $\phi$ (around 0.2) studied by the authors, some signatures of nonmonotonic behavior is found. But the data was fitted with a monotonic function, probably motivated by their earlier numerical study [21]. Furthermore, the apparent minimum observed in the numerical data for effective viscosity in Ref. [21] seems to occur at a half-integer value of the confinement parameter for one value of $\phi$ and seems to disappear at a higher value of $\phi$.

[25] D. Mitra, J. Bec, R. Pandit, and U. Frisch, Phys. Rev. Lett. 94, 194501 (2005).

[26] To take into account the movement of the spheres across layers, we calculate $r_{\|}$up to the time $(T)$ it takes for the wall-normal distance between the pair of spheres to become larger than $2 a$.

[27] M. Zurita-Gotor, J. Bławzdziewicz, and E. Wajnryb, Phys. Rev. Lett. 108, 068301 (2012).

[28] J. Katz, Phys. Rev. E 89, 021003 (2014).

[29] X. Xu, S. A. Rice, and A. R. Dinner, Proc. Natl. Acad. Sci. U.S.A. 110, 3771 (2013).

[30] M. Thiébaud, Z. Shen, J. Harting, and C. Misbah, Phys. Rev. Lett. 112, 238304 (2014).

[31] B. Kaoui, R. J. Jonk, and J. Harting, Soft Matter 10, 4735 (2014). 